\title{
Study of blood and its components by terahertz pulsed spectroscopy
}

\author{
O.P.Cherkasova $^{1,2}$, M. M. Nazarov ${ }^{3}$, A. P. Shkurinov ${ }^{4,5}$ \\ ${ }^{1}$ Institute of Laser Physics of SB RAS, Novosibirsk, Russia, o.p.cherkasova@ gmail.com \\ ${ }^{2}$ Tomsk State University, Tomsk, Russia \\ ${ }^{3}$ Kurchatov Institute National Research Center, Moscow, Russia \\ ${ }^{4}$ Crystallography and Photonics Federal Research Center, Russian Academy of Sciences, Moscow, Russia \\ ${ }^{5}$ Lomonosov Moscow State University, Moscow, Russia
}

Introduction. The prospect of widely using $\mathrm{THz}$ radiation in medical diagnosis and therapy raises the question of interaction of human tissues with $\mathrm{THz}$ radiation. Blood is a fluid connective tissue. Blood consists of plasma, and cellular elements. There are three main types of cellular blood cells: red blood cells (RBC), white blood cells (leukocytes) and blood platelets (platelets). Blood plasma contains $90 \%$ of water, about $6.6-8.5 \%$ of proteins and other organic and mineral compounds, which are intermediate or final products of metabolism, transferred from some organs in others. Capillaries with blood are in the skin and can be exposed to $\mathrm{THz}$ radiation. Therefore it is important to know the dielectric properties of blood and its components in $\mathrm{THz}$ range. The report will provide a review of the known data and our own studies on $\mathrm{THz}$ time domain spectroscopy (THz-TDS) of blood and its components at normal states and some pathology.

Experimental. The study of blood and its components is performed both in transmission and reflection configurations. Blood is poured into a special cuvette with a thickness (d) of 50-500 $\mu \mathrm{m}$. For $d<100$ $\mu \mathrm{m}$ thickness variation can introduce noticeable errors, especially in refraction index. In reflection spectroscopy, data variation is related to the time delay between the solution signal and the reference signal producing different slopes of the reflection spectrum phase. The results obtained by transmission spectroscopy are more reliable at lower frequencies $(0.05-0.8$ $\mathrm{THz}$ ), whereas reflection spectroscopy is preferable for higher $\mathrm{THz}$ frequencies $(1.0-2.5 \mathrm{THz})$ [1].

Results. $\mathrm{THz}$ absorption and reflection spectra of blood and its components have not any sharp spectral features (fig.1.) [1-3].

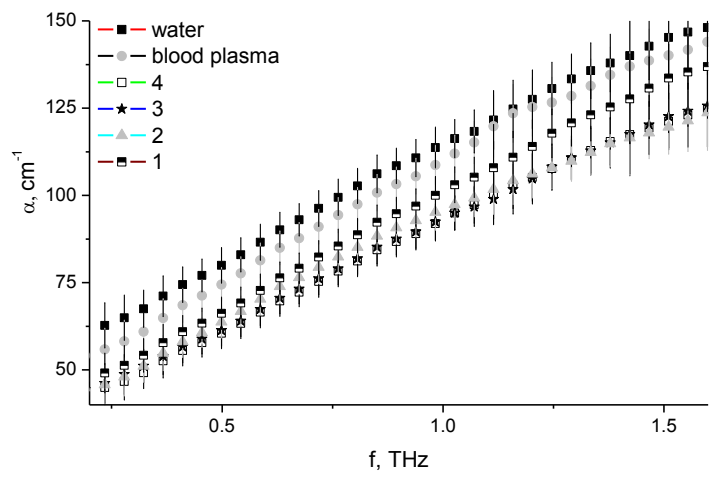

Fig. 1. Absorption coefficient of water, human blood plasma and RBC at different concentrations from $5.7 \cdot 10^{6}$ (1) to $7.32 \cdot 10^{6}(4)($ cells $/ \mathrm{ml})$.
The shape of the spectra corresponds to the form of water spectrum, and for analysis, it is possible to use the two-component Debye model [1-3]. It was shown that there are some distinction in the Debye relaxation coefficients for the whole blood and its components [2]. A significant difference can observe in both the absorption and refractive index spectra of the whole blood and its thrombus: the coagulation and the formation of a thrombus have affected the absorption coefficient as well as the Debye coefficients [2, 4]. It was demonstrated the capability to distinguish thrombus formation at its early stage from uncoagulated blood by the change in the absorption coefficient at 200-270 GHz [4].

It has been shown that the absorption coefficient decrease linearly with increasing RBC concentration in whole blood $[3,5,6]$. The excellent linearity between the $\mathrm{THz}$ signal and the RBC concentration was also confirmed in a polyurethane resin tube using a $\mathrm{THz}$ imaging method. These results demonstrate that THz-TDS imaging can facilitate the quantitative analysis of blood [5].

It has been demonstrated that various biological substances of blood have a significant effect on its optical properties. A clinical study of ex-vivo fresh human whole blood showed that triglycerides and the number of RBC were two dominant factors to have significant negative correlation to the sub-THz absorption coefficients. This circumstance should be taken into account for future $\mathrm{THz}$ investigation in human whole blood [6]. The effect of blood glucose concentration on the optical properties of whole human blood was shown [7]. By investigating the $\mathrm{THz}$ spectra of different human blood, it was found out $\mathrm{THz}$ absorption coefficients reflect a high sensitivity to the glucose level in blood. With a quantitative analysis of 70 patients, it was demonstrated that the $\mathrm{THz}$ absorption coefficients and the blood glucose levels have a linear relationship [8].

The reduction of the absorption of blood plasma from mice with experimental oncology (Ehrlich carcinoma) relatively that of healthy mice have been shown in paper [9]. The authors concluded that such components of whole blood as blood red cells and platelets make the major contribution to the blood absorption in the $\mathrm{THz}$ range and the changes in the composition of other components cannot be reliably detected using the methods of $\mathrm{THz}$ spectroscopy.

It has been shown that the absorption coefficient and the refractive index of diabetic rat blood plasma are small compared to those of water and plasma of healthy rats $[1,10]$. The diabetic rats have a higher 
level of glucose in blood and low protein concentration in comparison with healthy rats [10]. Assuming that the observed spectral changes are due to changes in the state of water in blood plasma, we have selected one of the parameters of the Debye model, $\Delta \varepsilon_{1} / \tau_{1}$, leading to the spectral features observed in the experiment. This change in the response of bound water can be the reason of the observed changes at increasing glucose concentration in blood plasma. We have demonstrated that when the concentration of glucose in blood rises to $24 \mathrm{mM}$ (in rats with diabetes), $\Delta \varepsilon_{1} / \tau_{1}$ ratio decreases in 1.2 times [1].

We carried out a detailed study of the influence of glucose and protein concentrations on $\mathrm{THz}$ response of water solutions at $0.05-3.2 \mathrm{THz}$ [11-13]. Different sensitivity to protein concentration in three spectral subregions, around $0.1,1$ and $3 \mathrm{THz}$ was shown, which can influence the quantitative analysis of blood components. We proposed a simple method for analyzing experimental data $[1,11-13]$

Conclusions. Whole blood and its components were studied by the THz-TDS method by a number of authors. The change of composition caused by pathological processes in the organism can considerably affect the optical properties of blood components in the $\mathrm{THz}$ frequency range. This can be used to create new rapid diagnostic methods. The cause of the detected differences was discussed with respect to variation in the terahertz response of water.

Acknowledgements. This work has been supported by Russian Foundation for Basic Research (project № 17-00-00275 (17-00-00270)).

\section{References}

1. Cherkasova, O.P., Nazarov, M. M., Angeluts, A.A., Shkurinov, A.P. Analysis of blood plasma at terahertz frequencies // Optics and Spectroscopy. 2016. V. 120(1). P. 50-57.

2. Reid, C.B., Reese, G, Gibson, A.P., Wallace, V.P. Terahertz time domain spectroscopy of human blood // IEEE J. Biomed. Health Inform. 2013. V. 3, No. 4. P. 363-367.

3. Angeluts, A.A., Balakin, A.V., Evdokimov, M.G. et. al. Characteristic responses of biological and nanoscale systems in the terahertz frequency range // Quantum Electronics. 2014. V. 44, No. 7. P. 614 632.

4. Sun, C. K., Chen, H. Y., Tseng, T.F. et. al., High Sensitivity of T-Ray for Thrombus Sensing // Scientific reports. 2018. V. 8:3948, DOI:10.1038/s41598018-22060-y

5. Jeong, K., Huh, Y.-M., Kim, S.-H., et.al. Characterization of blood using terahertz waves // J. Biomed. Opt. 2013. V. 18 (10), 107008.

6. Tseng T.-F., You, B, Gao H.-C., et. al. Pilot clinical study to investigate the human whole blood spectrum characteristics in the sub-THz region // Opt. Express. 2015. V. 23, No 7. P. 9440-9451.

7. Gusev,S.I., Demchenko,P.S., Cherkasova, O.P., Fedorov, V.I., Khodzitsky, M.K., Influence of glucose concentration on blood optical properties in $\mathrm{THz}$ fre- quency range // Chinese Optics. 2018. V. 11(2). P. 182-189.

8. Chen, H., Chen, X., Ma, S. et al. Quantify Glucose Level in Freshly Diabetic's Blood by Terahertz Time-Domain Spectroscopy // Journal of Infrared Millimeter and Terahertz Waves. 2018. V. 39. P. 399.

9. Smolianskaya, O.A., Kravtsenyuk, O.V., Panchenko, A.V. et al. Study of blood plasma optical properties in mice grafted with Ehrlich carcinoma in the frequency range $0.1-1.0 \mathrm{THz} / /$ Quantum Electronics. 2017. V. 47, No. 11. P. 1031 - 1040.

10. Cherkasova, O.P., Nazarov, M.M., Smirnova, I.N., Angeluts, A.A., Shkurinov, A.P. Application of Time-Domain $\mathrm{THz}$ Spectroscopy for Studying Blood Plasma of Rats with Experimental Diabetes // Physics of Wave Phenomena. 2014. V. 22, No. 3. P. 185-188.

11. Nazarov, M.M., Cherkasova, O.P., Shkurinov, A.P. Study of the dielectric function of aqueous solutions of glucose and albumin by $\mathrm{THz}$ time-domain spectroscopy // Quantum Electronics. 2016. V. 46, No 6. P. $488-495$.

12. Nazarov, M.M., Cherkasova, O.P., Shkurinov, A.P. Properties of aqueous solutions in $\mathrm{THz}$ frequency range // Journal of Physics: Conference Series. 2017. V. 793. Doi:10.1088/1742-6596/793/1/012005.

13. Nazarov, M. M., Cherkasova, O. P., Shkurinov, A. $P$. Comprehensive Study of Albumin Solutions in the Extended Terahertz Frequency Range // Journal of Infrared Millimeter and Terahertz Waves. 2018. V. 39 (9). P. 840-853. 